\title{
The Magnetic Field in the Stability of the Stars
}

\author{
Angel Fierros Palacios \\ Instituto de Investigaciones Eléctricas, División de Energías Alternas, Mexico City, México \\ Email: afierros@iie.org.mx
}

Received 4 June 2015; accepted 20 October 2015; published 23 October 2015

Copyright (C) 2015 by author and Scientific Research Publishing Inc.

This work is licensed under the Creative Commons Attribution International License (CC BY). http://creativecommons.org/licenses/by/4.0/

(c) (i) Open Access

\begin{abstract}
The theoretical frame developed by A. S. Eddington is used in this paper in order to study the problem of internal structure and stars stability. A new hypothesis is developed that consists of assume that at early stage of their evolution, all gaseous stars generate an intense magnetic field whose mission is to contribute to their stability. A simple methodology is shown to calculate the magnitude of the self-generated magnetic field at any point of the star, and diagrams are built for two specific cases, where the way in which this field varies from the center to the surface can be seen. Finally, the problem of the Cepheid type variables is studied obtaining an expression for the oscillation period carried out from the differential equation of the simple harmonic movement in terms of the appropriate parameters of the star.
\end{abstract}

\section{Keywords}

\section{Structure of Stars, Stability of the Stars, Cepheid Variables}

\section{Introduction}

The theory developed by Eddington considers the case of a star composed by a fluid which from the thermodynamic viewpoint behaves as an ideal gas. To determine the state of the material, two ordinary differential equations are proposed in order to mathematically express the following conditions [1].

The mechanical equilibrium of the star. In order to fulfil this condition, it is necessary that at any internal region of the star, the pressure has the right value in order to support the weight of the material above it; and The existence of thermal equilibrium. This condition requires that temperature distribution in the star is capable of maintaining itself automatically not with standing the continual transfer of heat from one part of the star to another.

The proposed equations are integrated and conditions of the material at any point are determined. This way 
the distribution of pressure, mass density and temperature are obtained. As work hypothesis, it is considered that at first approximation, the scheme distribution is homologous from star to star. This means that all gaseous stars copy the same stellar model within its appropriate scale of mass, length, temperature, etc. [1]. In order to, once and for all, simplify the task, a general solution to the problem is formulated and then the question is reduced to adapt it to the scale of the particular star being studied. It is important to notice that Eddington abandons J. Homer Lane's hypothesis [1] [2] when he does not consider that the thermal equilibrium in a gaseous star is due to the existence of internal convective streams. The hypothesis of convective equilibrium is replaced by the radiative equilibrium and this last basic principle is applied to the internal conditions of the sun and the stars [1].

In this work, a magnetic field as the fundamental element for the stars equilibrium will be introduced. It is proposed as a basic hypothesis, that all gaseous stars generate an intense magnetic field at an early stage of their evolution. Thus, a large mass of compressible gaseous fluid, viscous and conductor isolated in space and at very high temperature and pressure conditions that remain together by its own gravitational attraction and at dynamic equilibrium with the force produced by the sum of the pressures of radiation and the hot gases are considered. The source of power generation lies on the central part of this huge mass of gas and a little closer to the surface the so called convective zone [3] [4]. The convective streams generated there are responsible of removing the heat produced and the ashes of the thermo nuclear process, feeding at the same time the power source with new nuclear fuel [5]. It is assumed that these convective streams are made up by neutral atoms and a lot of electrically charged particles both positive and negative. It is also assumed that these convective streams contain a steady-state current distribution localized in some region inside the convective zone, produced by a process of maximum ionization, which by hypothesis is the generator of the magnetic field that in a first approximation, may be considered similar to the bipolar magnetic field produced by a magnetized bar. Irregularities observed in the magnetic field should be attributed to the fact that the structure of the localized steady-state current distribution is not always the same; this is because the condition of the convective streams is not always the same either, and can be dependent on the magnitude of the thermonuclear explosions produced in the oven. The solution of the problem of the magnitude and topology of the self-generated magnetic field by gaseous stars can be seen in Appendix II. It is significant to emphasize the importance that the self-generated magnetic field has for the equilibrium and the stability of this object. Additionally, this enormous concentration of matter is found distributed in a configuration that has spherical symmetry. A heavenly object with the above mentioned characteristics is a star.

It can be assured that at any point of the stellar fluid in equilibrium, there is a hydrostatic pressure which is the same in every direction. If a closed surface with in this configuration is drawn, the reaction of the external fluid to that surface over the internal fluid consists of a force per unit area exerted on the surface and along the inward normal. So that for the dynamic equilibrium existence, it is necessary for these surface forces to be balanced by body forces such as the gravitational which acts towards the inside of the star. It is also necessary to consider the contribution of the magnetic field. Its contribution to the state of equilibrium must be included in the theory in the way of a force per unit area known as the magnetic hydrostatic pressure [5]-[7]. As it is well known [8], a magnetic field has the property of generating in a conducting fluid, a magnetic viscosity that confers it to certain rigidity. That rigidity in the conducting stellar fluid may be interpreted as if the star were supported by a superstructure made up by the magnetic lines of force. It will be assumed in this paper that this magnetic superstructure has the mission of keeping the shape of the star even when this object should be animated by a rotation movement, since the magnetic lines of force are frozen in the stellar fluid and move along with it [5] [9].

\section{The Internal Structure and the Stability of a Gaseous Star}

In order to make an appropriate theoretical treatment of the problem of the stability of a star and of its internal structure, it is necessary to include the self-generated magnetic field in the fundamental equation that governs the state of equilibrium [1] which in this case is magneto mechanical [5], i.e.

$$
\frac{\mathrm{d}}{\mathrm{d} x}\left(p-\frac{H^{2}}{8 \pi}\right)=-\rho g
$$

where $p(x, t)$ is the whole pressure, $\rho(x, t)$ the mass density, $g$ the constant value of gravity acceleration and $H^{2} / 8 \pi$ the hydrostatic magnetic pressure, with $\boldsymbol{H}(x, t)$ being the magnetic field self-generated by the star [5]. 
The previous functions depend on time $t$ and of an $x$ distance measured from any internal region of the star to the center of it [1]. The other relationship that must be considered is the equation for the radiative equilibrium [1] [10]

$$
\frac{\mathrm{d} p_{r}}{\mathrm{~d} x}=-\frac{k \rho \varepsilon}{C} .
$$

In the previous formula $p_{r}(x, t)$ is the pressure of radiation, $c$ the velocity of light in the empty space, $\varepsilon$ the radiation energy by $\mathrm{cm}^{2}$ and by second and $k$ the coefficient which determines radiation absorption by stellar fluid [1]. From (1) and (2) it is easy to see that

$$
\mathrm{d} p_{r}=\left(\frac{k \varepsilon}{c g}\right) \mathrm{d}\left(p-\frac{H^{2}}{8 \pi}\right) .
$$

Let us consider a fluid mass distribution within the star contained in a radius sphere equal to $x$. In a stationary state, the amount of energy released per second within the sphere is equal to $L(x)$ and it is such that

$$
\varepsilon=\frac{L(x)}{4 \pi x^{2}}
$$

where $4 \pi \varepsilon x^{2}$ is the whole of the radiation that flows per second through the surface of the sphere and it is clearly equal to the amount of energy released by the central thermonuclear oven [1]. On the other hand, the gravitational force at the position $x$ is only due to the mass $M(x)$ which is found in the inside region to $x$ [11], so that

$$
g=\frac{G M(x)}{x^{2}} .
$$

With $G$ is the universal gravitational constant. From (4) and (5), the following result is obtained:

$$
\frac{\varepsilon}{g}=\frac{1}{4 \pi G} \frac{L(x)}{M(x)}
$$

where $L(x) / M(x)$ is the average rate of energy release per gram from the inner region to $x$ [1]. It is assumed that this energy release is greater in the dense center of the star than in its external parts, in such way that this reason decreases as $x$ is increased when succeeding layers of colder material are added to the average [1].

Be $M$ the total mass of the star and $L$ the total emission of energy per second from its surface in such way that $L / M$ is the boundary value that reaches the reason $L(x) / M(x)$. For all of the above mentioned it is possible to write the following relation [1]

$$
\frac{L(x)}{M(x)}=\eta \frac{L}{M} .
$$

There are reasons to state that $\eta$ is a magnitude which is increased from 1 on the star surface up to an unknown value although not very big in the center of it [1]. Its analytical form depends on the law of energy release by the thermonuclear oven [1]. Nevertheless, it is possible to assume that this law is approximately of the same kind for all the stars since presumably the nuclear energy releasing mechanism is basically the same for all of them [1]. If (7) is substituted in (3) the following result is obtained

$$
\mathrm{d} p_{r}=\frac{L \eta k}{4 \pi G c M} \mathrm{~d}\left(p-\frac{H^{2}}{8 \pi}\right) .
$$

This last relation is an exact equation which allows establishing an upper limit for the value of the opacity for any star for which magnitudes $L$ and $M$ are known as a result of observation, regardless of whether the star is or not constituted by a fluid obeying the thermal equation of perfect gas [1].

In any case it is expected that the values of temperature and mass density are increased towards the inner part of the star [1]. Consider that the whole pressure $p$ is such that [1]

$$
p=p_{g}+p_{r}
$$


where $p_{g}(x, t)$ is the pressure of hot gases. As on the other hand it is clear that

$$
\mathrm{d}\left(p-\frac{H^{2}}{8 \pi}\right)=\mathrm{d} p_{r}+\mathrm{d}\left(p_{g}-\frac{H^{2}}{8 \pi}\right)
$$

in (8) the following fundamental relation is obtained

$$
\mathrm{d} p_{r}=\frac{L \eta k}{4 \pi G c M-L \eta k} \mathrm{~d}\left(p_{g}-\frac{H^{2}}{8 \pi}\right) .
$$

Since the tendency of temperature and mass density is that of increasing its values towards the inner part of the star, it can be stated that the pressure of the material $p_{g}$ and the magnetic hydrostatic pressure $H^{2} / 8 \pi$ should increase their intensities so that the stability of the star is kept. Under these conditions the following inequality is expected to be fulfilled

$$
\mathrm{d} p_{r}<\mathrm{d}\left(p_{g}-\frac{H^{2}}{8 \pi}\right)
$$

According to (1)

$$
\frac{L \eta k}{4 \pi G c M-L \eta k}<1 .
$$

Since $\eta$ is always positive [1] we have that $\eta>1$, in such way that from the previous relation the following is obtained

$$
k<2 \pi G c M / L
$$

that with the constants numerical values is transformed into

$$
k<12500 \frac{M}{L} .
$$

The previous numerical factor is only half of what is reported in the specialized literature [1]. For the most brilliant component of Capella's binary system we have the following basic data:

$$
\begin{aligned}
& M=8.3 \times 10^{33} \mathrm{gr} \\
& L=4.8 \times 10^{35} \mathrm{ergs} \cdot \mathrm{seg}^{-1} .
\end{aligned}
$$

In that case

$$
k<216 \mathrm{~cm}^{2} \cdot \mathrm{gr}^{-1} .
$$

For the Sun we have that $k<6564 \mathrm{~cm}^{2} \cdot \mathrm{gr}^{-1}$. The physical reason for the existence of that upper limit known by the name of Eddington limit [1] [10] is the following. So that the radiation is observed has to be emitted reaching its way through the star, and if there were too much obstruction it would blow up the star [1]. However, the existence of the self-generated magnetic field is a determining factor for the star to keep its stability. In fact, the superstructure built by the magnetic lines of force is a dynamic obstacle which prevents the star from collapsing because of gravitational compression or exploding because of the added effect of the radiation pressures and of the hot gases. Ultimately, the role played by the magnetic hydrostatic pressure in this part of the theory is that of diminishing to a half the limit value of $k$.

This fact is important since it indicates that in general gaseous stars must be more luminous than it is believed and therefore that their age can be less than that which has been determined by Eddington's theory [12]. In specialized literature [12] it is stated that the age of stars is inversely proportional to luminosity [12]. Since luminosity is inversely proportional to opacity [1] as it will be seen further on, if the value of the Eddington limit of the opacity is in fact smaller than the one calculated so far, luminosity will be greater and therefore the age of the stars will be smaller.

Incidentally, an increase in luminosity is indicative of the fact that the star will live less since it is burning its fuel at a greater rate. With this result the apparent paradox of the stars which are older than the Universe itself, can be solved. In conclusion and within the present theoretic scheme, it can be stated that the age of gaseous stars is ruled by the modified Eddington limit of the coefficient of opacity. 


\section{The Magnetic Field on the Surface of a Star}

On the surface of any gaseous star the magnitude of the self-generated magnetic field follows a very simple law as we shall see next. Its absolute value can be determined from the condition of magneto mechanical equilibrium (1) and considering that $\rho=\rho(R, t)$, with $R$ the star's radius. When that relation is integrated it is easy to obtain the following result [5]

$$
p-\frac{H^{2}}{8 \pi}=\rho \boldsymbol{g} \cdot \boldsymbol{x}+\text { constant } .
$$

On the other hand, it is known that the pressure of hot gases $p_{g}(x, t)$ satisfies the hydrostatic equation [5] [13], i.e.

$$
p_{g}(\boldsymbol{x}, t)=\rho \boldsymbol{g} \cdot \boldsymbol{x}+\text { constant } .
$$

In that case and with the help of the relation (9) it can be proved that

$$
p_{r}=\frac{H^{2}}{8 \pi} .
$$

The preceding formula must be considered as a relation of equivalence more than an equality. From it and with the help of the expression for the pressure of the radiation, the absolute value of the magnetic field can be calculated. Since for a perfect gas it is fulfilled that

$$
p_{r}=\frac{1}{3} a T^{4}
$$

where $a=7.64 \times 10^{-15}$ is Stefan's constant [1], of (18) it can be proved that

$$
H=m T_{e}^{2}
$$

with

$$
m=\left[\frac{8 \pi a}{3}\right]^{1 / 2}
$$

a universal constant that has the following numerical value

$$
m=2.53 \times 10^{-7} \text { gauss } \cdot \mathrm{K}^{-2} \text {. }
$$

The relation (20) allows calculating the absolute value of the average magnetic field on the surface of a any gaseous star if its effective temperature $T_{e}$ is known. The effective temperature can be calculated from the radiation theory of the black body [1] [14]. If $\mathrm{E}$ is the energy density radiated by the black body, it can be shown that

$$
\mathrm{E}=a T^{4} \text {. }
$$

The former result is known as the name of Stefan's law [1]. Since for a perfect gas $\mathrm{E}=3 p_{r}$ [1], the relation (19) is satisfied. On the other hand, the radiation emitted by a radius sphere $r$ every second is [1]

$$
L=\left(4 \pi r^{2}\right)\left(\frac{a c T_{e}^{4}}{4}\right)=\pi a c r^{2} T_{e}^{4} .
$$

Therefore, the effective temperature of a star is defined by the following relation [1] [10]

$$
T_{e}=\left[\frac{L}{\pi a c R^{2}}\right]^{1 / 4} .
$$

since it gives the black body temperature which produces the same amount of radiation emitted by the star [1]. In (24) and (25) $L$ is the luminosity. It is important to clarify that the effective temperature is a conventional measurement that specifies the reason of radiant flux heat per unit area. It must not be considered as the temperature at any particularly significant level in the star [1] [10] [12]. Finally, it is known that the temperature of the photosphere of the Sun is equal $5.741 \times 10^{3} \mathrm{~K}$. Consequently $H_{\odot}=8.4$ gauss on its surface. Another example is 
that of the most brilliant component of Capella's binary system for which it is known that its effective temperature is equal to $5.2 \times 10^{3} \mathrm{~K}[1]$. In that case $H_{c}=6.8$ gauss.

\section{The Mass-Luminosity Relation and the Opacity Coefficient}

Let us suppose that in the Equation (11) the product $\eta k$ is a constant through the star. In order to support that hypothesis it is required that the absorption coefficient $k$ be practically constant decreasing a little towards the center to counterbalance the increase of $\eta$, in that way assuring that the product of these two quantities remains constant [1] [10] [12]. It is said that there are very good reasons to believe that in general $k$ behaves in this manner [1], wherefore it is possible to state that the constancy of this product is a good approximation [1]. Be therefore

$$
\eta k=k_{o}
$$

with $k_{o}$ a constant that somehow represents the boundary value of $k$ [1]. It is important to emphasize that the value of $k$ in the stellar photosphere could be very different from the one that it would have there $k_{o}$ [1]. Using the approximation (26) in (11) and integrating, the following result is obtained

$$
p_{r}=\frac{L k_{o}}{4 \pi G c M-L k_{o}}\left(p_{g}-\frac{H^{2}}{8 \pi}\right)
$$

that with the help of the relation (18) is transformed into what follows

$$
p_{r}=\frac{L k_{o}}{4 \pi G c M} p_{g} .
$$

Clearly, it fulfills the fact that for a particular star the ratio $p_{r} / p_{g}$ always maintains the same relation. Consequently

$$
\frac{L}{M}=\frac{4 \pi G c}{k_{o}}\left(\frac{p_{r}}{p_{g}}\right) .
$$

At this moment it is important to introduce a constant $\beta$ defined as follows [1]

$$
\begin{aligned}
& p_{r}=(1-\beta) p \\
& p_{g}=\beta p ;
\end{aligned}
$$

where $p$ is whole pressure. $\beta$ represents the ratio between hot gas pressure and whole pressure, whilst $(1-\beta)$ is the ratio between radiation pressure and whole pressure. Therefore, the ratio $(1-\beta) / \beta$ can be considered as a measurement of the degree of stability of a star. Substituting (30) in (29) we have that

$$
\frac{L}{M}=\frac{4 \pi G c}{k_{o}}\left(\frac{1-\beta}{\beta}\right) \text {. }
$$

For gaseous stars the value of $\beta$ is determined from the following quadric equation [1]

$$
1-\beta=3.09 \times 10^{-3}(M / \odot)^{2} \mu^{4} \beta^{4}
$$

where the symbol $\odot$ indicates the mass of the Sun and $\mu$ the average molecular weight [1] [10]. The relation (32) can be solved for various values of mass $M$ in terms of the sun's mass, e.g. $M=1 / 4,1 / 2,1, \ldots$ times $\odot$; and also for some average value of $\mu$. It is important to point out that $\beta$ only depends on the mass and average molecular weight of the material forming the star and it is independent of its radius and its opacity [1]. Once obtained the value of $\beta$ by solving the Equation (32) it is possible to determine the opacity $k_{o}$ from the relation (31). In specialized literature [1] there are tables which can be consulted to use in numerical calculations which contain values from $(1-\beta)$ for different masses and molecular weights.

\section{Luminosity and Opacity}

If the possible small changes of $\mu$ with the temperature and density [1] are neglected, it is possible to see from (32) that $(1-\beta)$ is a function only of the mass of the star. In that case and according to (31) it can be stated that 
for gaseous stars of the same mass, the luminosity is inversely proportional to the opacity $k_{o}$ [1].

However the approximate constancy of $k_{o}$ from star to star should be distinguished from the approximate constancy of the product $\eta k$ within a single star [1]. According to theoretical analysis carried out by other researchers [1] [10] [12], it can be stated that for a particular star the following relation is satisfied [1]

$$
\eta T^{-1 / 2}=\frac{\eta k}{k_{c}}=\frac{k_{o}}{k_{c}}
$$

where $k_{c}$ refers to density and temperature conditions in the center of the star. On the other hand, the law of absorption of the radiation commonly accepted [1] [10] [12] has the following analytical form

$$
k=k_{1} \rho T^{-7 / 2}
$$

with $k_{1}$ is a constant. In that case it is customary to write that [1]

$$
k_{o}=\alpha k_{c}
$$

where $\alpha$ is a constant that according to (33) is equal to

$$
\alpha=\eta T^{-1 / 2} .
$$

The constancy of $\eta T^{-1 / 2}$ depends on the ratio of energy release; release which is likewise related to the distribution of energy sources in the inner part of the star. What it normally does is to consider various degrees of concentration of those sources and to examine how the ratio of energy release per gram, $\varepsilon$, for different temperature powers varies [1] [10]. However, even when the constancy of that factor is better for $\varepsilon \sim T$, it is customary to explore other possibilities [1]. For cases in which $\eta T^{1 / 2}$ is not absolutely constant, the best approximation to the ratio $k_{o} / k_{c}$ may be obtained from the average. From specialized literature [1] we have that

$$
\begin{array}{rlrrr}
a & =1.32 & 1.74 & 2.12 & 2.75 \\
\text { for } \varepsilon & \sim \text { constant } & T & T^{2} & T^{4}
\end{array}
$$

respectively. It is said that when the sources are very concentrated in the center of the star brightness diminishes. In the case in which numerical calculations are required, the value $\alpha=2.5$ is adopted, which is indicative of an intense and uniform concentration of sources of energy release in the center of the star [1]. Consequently in (31) the modified mass-luminosity relation is obtained

$$
L=\frac{4 \pi G c M}{\alpha k_{c}} \cdot\left(\frac{1-\beta}{\beta}\right) .
$$

When numerical calculations are made it is customary to assume that $k_{c}=k$ [1].

\section{The Central Temperature}

In order to calculate the central temperature in any gaseous star, relations (16) and (17) are used as well as the fact that the relation of equivalence (18) is always fulfilled as much on the surface as in any inner region of the star. Since for gaseous stars the thermal equation of ideal gas is fulfilled [1] [10] [15]

$$
p_{g}=\frac{R \rho T}{\mu}
$$

where $R=R_{o} / m_{H}$ is the gases universal constant [1] [15], $R_{o}$ is Boltzmann's constant, $m_{H}$ hydrogen atom mass and $\mu$ the molecular weight which is generally taken as a constant numerically equal to 2.2 [1] [10] [12], of (9) and from relations (30) the following result is obtained

$$
\frac{T^{3}}{\rho}=\frac{3 R(1-\beta)}{a \mu \beta}
$$

The previous relation is equivalent to the equation

$$
T^{3} / \rho=1.53 \times 10^{18} k_{c} L / M
$$

where results (38) and (40) were used. 
In general, ratio $T^{3} / \rho$ which is a constant through any star is also the same for all the stars that have the same mass; whenever it is possible small differences in the average molecular weight $\mu$ that may exist among them, should be ignored [1] [10] [12]. Consequently in stars of the same mass, the temperature at homologous points in the interior varies as the cube root of mean density measured at these points does [1] [10]. As it is easy to see from paragraph 2, effective temperature is subject to a different law.

\section{The Problem of Variable Stars of the Cepheid Type}

The theory commonly accepted about the variable stars of the Cepheid type [1] [10] attributes the variation in their brightness to a regular pulsation they experiment.

In order to make an adequate theoretical analysis of the problem, it will be assumed that they are gaseous objects where the self-generated magnetic field has lost much of its original intensity but, that in the position of maximum compression keeps enough of it, so as to reduce the oscillation preventing the final collapse. Next, and with the help of radiation pressure and that of hot gases, that diminished magnetic field bounces, starting the subsequent expansion. If it is admitted that the Cepheids are in a stage of their evolution such that the fluid that forms them is no longer totally supported by the superstructure formed by the magnetic lines of force, it can be stated that the important dynamic agents acting on them are the huge gravitational force as well as the combined pressures of radiation and hot gases. Subject to these dynamic conditions, the Cepheids variables oscillate around some equilibrium position losing and recovering brightness alternately, as they expand and contract themselves with a noticeable regularity and with a perfectly determined period.

In accordance to the laws of gases, when the star collapses because of the effect of its huge weight and reaching even an extreme position or minimum size, the gaseous fluid heats up and the brightness of the Cepheid is increased. Right after that the hot gases, the radiation pressure and the residual magnetic field which behaves as a spring, act against gravity distending the star as far as another extreme position of maximum amplitude, causing the stellar fluid to cool and the Cepheid to lose brightness.

The cycle is repeated once and once again with regularity very much alike that observed in the movement of a simple harmonic oscillator [15]. To obtain the differential equation governing the phenomenon and also an expression for the period of harmonic oscillation, Equations (11), (18) and (26) must be considered in order to obtain the following relation:

$$
\mathrm{d} p_{r}=\frac{L k_{o}}{4 \pi G c M} \mathrm{~d} p_{g}
$$

Integrating the above equation the following is obtained

$$
p_{r}+p_{r}^{o}=\frac{L k_{o}}{4 \pi G c M}\left(p_{g}+p_{g}^{o}\right)
$$

where $p_{r}^{o}$ and $p_{g}^{o}$ are integration constants with unities of force per unit area. Besides $p_{g}$ satisfied the hydrostatic Equation (17). Let us now consider a radius sphere $r$ concentric with the star and with its surface almost coinciding with that of the star in such a way that the amount of mass $M(r)$ contained in it is practically equal to the total mass $M$ of the star. Under these conditions, the gravity acceleration on the surface of the star is

$$
g \approx \frac{G M}{r^{2}} .
$$

It is proposed that as much on the surface of the sphere as on that of the star, $p_{g}^{o}$ and the constant in (17) be equal to zero. However, there is $p_{r}^{o} \neq 0$ because the pressure of radiation does not disappear on the surface [1]. According to the first of the definitions (30), in (43) the following result is obtained

$$
(1-\beta) p+p_{r}^{o}=-\frac{L k_{o} \rho}{4 \pi c r}
$$

where $p$ is whole pressure such that

$$
p=\frac{f}{4 \pi R^{2}}
$$

with $f$ the magnitude of total force, $4 \pi R^{2}$ the area of the surface of the star and $R$ its radius. Be 


$$
p_{r}^{o}=\frac{f}{4 \pi r^{2}}
$$

in such way that in (44) the following is obtained

$$
f\left[1-\beta+\frac{R^{2}}{r^{2}}\right]=-\frac{L k_{o} \rho}{c} \frac{R^{2}}{r} .
$$

In $r=R$ we have that

$$
f=-\frac{L k_{o} \rho}{c(2-\beta)} r .
$$

According to Newton's second law, of (47) the following result is obtained [15]

$$
M \ddot{r}+K r=0
$$

where $\ddot{r}=\mathrm{d}^{2} r / \mathrm{d} t^{2}$. The above relation is the differential equation that governs the harmonic oscillations observed on the Cepheid variable stars. In it,

$$
K=\frac{L k_{o} \rho}{c(2-\beta)}
$$

is a constant which depends on some basic parameters of the particular star being studied. The oscillations period is given by the relation $\tau=2 \pi(M / K)^{1 / 2}$, namely

$$
\tau=6.28 \times\left[\frac{M c(2-\beta)}{L \alpha k_{c} \rho_{m}}\right]^{1 / 2}
$$

where the relation (35) was used. Since $M$ and $L$ are total mass and luminosity respectively, in (50) average density $\rho_{m}$ must be used. From the mass-luminosity relation (38) and given that $4 \pi G=8.3 \times 10^{-7} \mathrm{ergs}^{\cdot} \mathrm{cm}^{\cdot \mathrm{gr}^{-2}}$, it is easy to see that

$$
\rho_{m}^{1 / 2} \tau(\text { days })=0.08 \times\left[\frac{\beta(2-\beta)}{1-\beta}\right]^{1 / 2} .
$$

As it was to be expected [3] [16], the product of the period in days and the square root of the mean density is equal to a constant that can be calculated from the theory. Next the periods of three known Cepheids are calculated and each result is compared to the one measured by direct observation.

1. $\delta$ Cepheid

For this intrinsic variable there are the following data (See Appendix)

$$
\begin{aligned}
& \rho_{m}=3.5 \times 10^{-4} \mathrm{gr} \cdot \mathrm{cm}^{-3} \\
& \beta=0.545 \\
& 1-\beta=0.455 \\
& 2-\beta=1.455
\end{aligned}
$$

Then,

$$
\tau=5.61 \text { days . }
$$

The period directly measured is of 5.366 days. As it is easy to see, theoretical calculation and direct measuring are practically equal.

2. Polaris

For this case we have that [1]

$$
\begin{aligned}
& \rho_{m}=8 \times 10^{-4} \mathrm{gr} \cdot \mathrm{cm}^{-3} \\
& \beta=0.58 \\
& 1-\beta=0.42 \\
& 2-\beta=1.42
\end{aligned}
$$


Therefore,

$$
\tau=3.97 \text { days . }
$$

The period measured for this variable is equal to 3.968 days.

3. $\beta$ Cepheid

The data we have are as follows [1]

$$
\begin{aligned}
& \rho_{m}=1.05 \mathrm{gr} \cdot \mathrm{cm}^{-3} \\
& \beta=0.67 \\
& 1-\beta=0.33 \\
& 2-\beta=1.33
\end{aligned}
$$

Consequently, $\tau=0.127$ days ; while $\tau$ (measured) $=0.190$ days. It is possible that discrepancies observed in the two last mentioned cases are due to the fact that there are not more accurate data for these stars. However, the magnitude order is adequate.

The long-period variables have, with some exceptions, periods ranging from 100 to 500 days, with a strong preference for periods near 300 days. The periodicity in not perfect and the stars may be some weeks behind or ahead of the predicted phase, the amplitude of the variation is not always the same in successive periods. Nevertheless, there is enough evidence that long-period variation and Cepheid variation are essentially the same phenomenon. The very low mass density and temperatures of the long-period variables exaggerates and renders more erratic the effects of the same kinds of pulsation as in the Cepheids [1]. In general, the longer the period of a red variable, the less regular is its pulsation; as in the cases of Betelgeuse and Mira. So, for that class of variables the period-mass density relation is

$$
\tau \rho_{m}^{1 / 2}=0.24 \times\left[\frac{\beta(2-\beta)}{1-\beta}\right]^{1 / 2} .
$$

This is so, because if $1 \mathrm{sec}=0.1157 \times 10^{-4}$ days, and the period of the red variable star which will be use as the unit measure is equal to $3 \times 10^{2}$ days, it has that

$$
0.1157 \times 10^{-4} \times 3 \times 10^{2}=0.347 \times 10^{-2} \text {. }
$$

Hence from (50), $6.87 \times 10^{3} \times 0.347 \times 10^{-2}=24$.Then, and given that the long-period variation and Cepheid variation are the same phenomenon, it is fulfilled that

$$
24 \times\left[\frac{\beta(2-\beta)}{1-\beta}\right]^{1 / 2}=0.08 \times 3 \times 10^{2} \times\left[\frac{\beta(2-\beta)}{1-\beta}\right]^{1 / 2}
$$

in such o way that $0.24=0.08 \times 3$. It is easy to see that is enough to multiply the numerical factor of the relation (51) by 3 , in orden to obtain (52). Next, the results for the red variable Betelgeuse are given:

\section{Betelgeuse}

\begin{tabular}{cc}
$\tau=3.65 \times 10^{2}$ days & $\rho_{m}=4.5 \times 10^{-7} \mathrm{gr} \cdot \mathrm{cm}^{-3}$ \\
$\tau \rho_{m}^{1 / 2}=0.245$ & $\rho_{m}^{1 / 2}=0.67 \times 10^{-3}$ \\
$\beta=0.392$ & $M=4.1 \times 10^{34} \mathrm{gr}$ \\
$1-\beta=0.608$ & $R=3 \times 10^{13} \mathrm{~cm}$ \\
$T_{e}=3 \times 10^{3} \mathrm{~K}$ & $\mu=2.11$ \\
$H_{s}=2.28$ gauss & $M_{\text {bol }}=-5.5$ \\
$M_{\text {vis }}=+0.41$ & \\
\hline
\end{tabular}

$r=150$ parsecs $=500$ light-years. 
Omicron Ceti, also called Mira, is a red variable star which has a period equal to $3.3 \times 10^{2}$ days, that is to say, its period is less that the one of Betelgeuse and hence, its average mass density must be greater than the Betelgeuse's average mass density. In consequence and given that $\tau_{0}=0.9 \tau_{B}$, it follows that $\rho_{m o}=1.11 \rho_{m B}$; and then, for the case of Omicron Ceti the following results are obtained.

\section{Omicron Ceti}

\begin{tabular}{cc}
$\tau=3.3 \times 10^{2}$ days & $\rho_{m}=5.4 \times 10^{-7} \mathrm{gr} \cdot \mathrm{cm}^{-3}$ \\
$\tau \rho_{m}^{1 / 2}=0.244$ & $\rho_{m}^{1 / 2}=0.74 \times 10^{-3}$ \\
$\beta=0.39$ & $M=4.1 \times 10^{34} \mathrm{gr}$ \\
$1-\beta=0.61$ & $R=2.8 \times 10^{13} \mathrm{~cm}$ \\
$T_{e}=2.6 \times 10^{3} \mathrm{~K}$ & $\mu=2.11$ \\
$M_{v \mathrm{sis}}=-0.45$ & $M_{\text {bol }}=-4.7$ \\
\hline
\end{tabular}

$r=70$ parsecs $=230$ light-years .

\section{The Magnetic Field in the Inner Part of a Gaseous Star}

Just as temperature follows different laws on the surface and in the inner part of a star, something similar happens to the self-generated magnetic field. On the surface of the star its behavior is ruled by the relation (20) whereas in the inner part of it, it follows another different law as it will next be seen. In order to calculate the magnitude of the magnetic field at the center of the star as well as at any other inner point of it, it is necessary to use the polytropic gas sphere theory [1] [10]. In terms of the gravitational potential $\phi(x)$, the acceleration of gravity is by definition [1]

$$
g=-\frac{\mathrm{d} \phi}{\mathrm{d} x} .
$$

Be $P=p-H^{2} / 8 \pi$, in such a way that in (1) the following result is obtained

$$
\mathrm{d} P=-g \rho \mathrm{d} x
$$

that with the aid of (53) is transformed into what follows

$$
\mathrm{d} P=\rho \mathrm{d} \phi .
$$

From Poisson's equation for the gravitational potential [17]

$$
\nabla^{2} \phi=-4 \pi G \rho
$$

with $\nabla^{2}=\operatorname{div}$ grad the laplacian operator [9], we have that for spherical symmetry the above relation takes the following form,

$$
\frac{\mathrm{d}^{2} \phi}{\mathrm{d} x^{2}}+\frac{2}{x} \frac{\mathrm{d} \phi}{\mathrm{d} x}=-4 \pi G \rho
$$

Now we have the relations (54) and (55) in order to be able to determine the three following unknown functions of $x: P, \rho \mathrm{y} \phi$. Then, a third equation is also required in order to take into account the thermodynamic state of the star. In general and regardless of whether the stellar gas is perfect or not, it is always possible to make any value of $P$ correspond to a given mass density if temperature is adequately fixed. What is usual [1] is to use as a third relation the following

$$
P=\kappa \rho^{\gamma}
$$

where $\kappa$ and $\gamma$ are disposable constants. Thus and for different values of $\kappa$ and $\gamma$ it is possible to investigate a va- 
riety of temperature distributions. It is said that distribution is polytropic [1] if it obeys an equation such as (56). The problem is reduced to redoing the analysis carried out by Eddington [1], task that will not be repeated in this paper. However, and following that methodology, we have that the whole pressure $p$ in terms of gravitational potential has the following

$$
p=\frac{\rho \phi}{(n+1) \beta}
$$

where $n$ is a positive integer number. Given that the thermal equation of the state of the ideal gas (39) is fulfilled, from (57) we have that

$$
T=\frac{\mu \phi}{R(n+1)} .
$$

As it is easy to see, $T=$ constant $\phi$. Be $\phi_{o}$ the gravitational potential in the center of the sphere and $T_{o}$ the central temperature so that $T_{o}=$ constant $\phi_{o}$; where the constant is the same as before. It can be seen that according to (58) and with the results of specialized literature [1], it is easy to see that for a particular star

$$
T_{o}=\frac{\mu}{R(n+1)} \cdot \frac{G M}{M^{\prime}} \cdot \frac{R^{\prime}}{R}
$$

where $M^{\prime}$ y $R^{\prime}$ are parameters calculated from the polytropic gas sphere theory [1] and

$$
\phi_{o}=\frac{G M}{M^{\prime}} \frac{R^{\prime}}{R} .
$$

The relation (59) is used to calculate the central temperature of any gaseous star for which its radius and mass are known [1]. Apart from this and according to the first of the relations (30), from (57) the following result is obtained

$$
p_{r}=\frac{\rho \phi(1-\beta)}{(n+1) \beta} .
$$

For gaseous stars the relation (56) takes the following form [1]

$$
P \approx \rho^{4 / 3}
$$

in such a way that $n=3$ [1]. With this result and with the relation of equivalence (18), it is easy to see from (58) that for a particular star

$$
\frac{H^{2}}{\rho \phi}=\text { constant }
$$

where

$$
\text { constant }=2 \pi\left(\frac{1-\beta}{\beta}\right)
$$

Consequently, for stars of the same mass as well as for homologous points in the inner part of a given star, the self-generated magnetic field varies like the square root of the product of the mass density and the gravitational potential; both calculated at those points.

For any inner region of the star, the magnitude of the self-generated magnetic field can be calculated from (63), namely

$$
H=\left[2 \pi \rho \phi\left(\frac{1-\beta}{\beta}\right)\right]^{1 / 2} .
$$

It is customary for numerical calculations to use the values given in Table 1 [1].

Usually the problem is reduced to finding the internal distribution for the density as well as for the pressure, in a star for which its mass and its radius or its mean density are known. The expressions used are the following [1]. 
Table 1. $(\mathrm{n}=3, \gamma=1.3333)$.

\begin{tabular}{|c|c|c|c|c|c|c|}
\hline$z$ & $u$ & $u^{n}$ & $u^{n+1}$ & $-\mathrm{d} u / \mathrm{d} z$ & $-z \mathrm{dz} / 3 \mathrm{~d} u$ & $-z^{2} \mathrm{~d} u / \mathrm{d} z$ \\
\hline 0.00 & 1.00000 & 1.00000 & 1.00000 & .00000 & 1.0000 & 0.0000 \\
\hline 0.25 & 0.98975 & 0.96960 & 0.95966 & 0.08204 & 10.0158 & 0.0051 \\
\hline 0.50 & 0.95987 & 0.88436 & 0.84886 & 0.15495 & 1.0756 & 0.0387 \\
\hline 0.75 & 0.91355 & 0.76242 & 0.69650 & 0.21270 & 1.1754 & 0.1196 \\
\hline 1.00 & 0.85505 & 0.62513 & 0.53451 & 0.25219 & 1.3218 & 0.2522 \\
\hline 1.25 & 0.78897 & 0.49111 & 0.38747 & 0.27370 & 1.5224 & 0.4276 \\
\hline 1.50 & 0.71948 & 0.37244 & 0.26797 & 0.27993 & 1.7862 & 0.6298 \\
\hline 1.75 & 0.64996 & 0.27458 & 0.17847 & 0.27460 & 2.1213 & 0.8110 \\
\hline 2.00 & 0.58282 & 0.19796 & 0.11538 & 0.26149 & 2.5195 & 1.0450 \\
\hline 2.50 & 0.46109 & 0.09803 & 0.04520 & 0.22396 & 3.7210 & 1.3994 \\
\hline 3.00 & 0.35921 & 0.04635 & 0.01665 & 0.18393 & 5.4370 & 1.6553 \\
\hline 3.50 & 0.27629 & 0.02109 & 0.005828 & 0.14859 & 7.8697 & 1.8203 \\
\hline 4.00 & 0.20942 & 0.009185 & 0.001923 & 0.11998 & 11.113 & 1.9197 \\
\hline 4.50 & 0.15529 & 0.003746 & 0.000582 & 0.09748 & 15.387 & 1.9740 \\
\hline 5.00 & 0.11110 & 0.001371 & 0.000152 & 0.08003 & 20.826 & 2.0007 \\
\hline 6.00 & 0.04411 & 0.000086 & 0.000004 & 0.05599 & 35.720 & 2.0156 \\
\hline 6.80 & 0.00471 & 0.000001 & 0.000000 & 0.04360 & 51.987 & 2.0161 \\
\hline 6.9011 & 0.00000 & 0.000000 & 0.000000 & 0.04231 & 54.360 & 2.0150 \\
\hline
\end{tabular}

$$
R^{\prime}=(z)_{u=0} ; \quad M^{\prime}=\left(-z^{2} \frac{\mathrm{d} u}{\mathrm{~d} z}\right)_{u=0} .
$$

The condition $u=0$ indicates the boundary of the star, whereas the numerical values of the parameters $R^{\prime}$ and $M^{\prime}$ may be consulted in the last line of the table. Another important relation is the one for the ratio between the mean and central densities

$$
\frac{\rho_{m}}{\rho_{o}}=\left(-\frac{3}{z} \frac{\mathrm{d} u}{\mathrm{~d} z}\right)_{u=0} .
$$

Its numerical value can be found at the bottom of the sixth column. On the other hand and since $\phi / \phi_{o}=u$, it is easy to see that [1]

$$
\frac{T}{T_{o}}=u
$$

where $T_{o}$ is the central temperature which can be calculated from the relation (58) for a constant value of the average molecular weight $\mu$ equal to 2.2 [1]. The numerical value of $u$ can be looked up on the second column of the table for different points within the star. For density we have the following relation [1]

$$
\frac{\rho}{\rho_{o}}=u^{n} .
$$

With $\rho$ is the density at some inner region of the star. It is calculated from $\rho_{o}$ and using the numerical values of $u^{n}$ for different points within the star which are recorded on the third column. In order to show how the formulas above and the table should be used, consider as the first example that of Capella's brightest component. 
Amongst the many data known of her we have that $M=8.3 \times 10^{33}$ gr and $R=9.55 \times 10^{11} \mathrm{~cm}$. Its mean density is $\rho_{m}=0.00227$ and in column sixth it can be seen that the ratio $\rho_{o} / \rho_{m}$ is equal to 54.36; so that in the center of the star

$$
\rho_{o}=0.1234 \mathrm{gr} \cdot \mathrm{cm}^{-3} \text {. }
$$

Also from the last line of the table the following values are obtained

$$
\begin{aligned}
& M^{\prime}=2.015 \\
& R^{\prime}=6.901
\end{aligned} \text {. }
$$

According to (60) the gravitational potential in the center is [1]

$$
\phi_{o}=\frac{6.66 \times 10^{-8} \times 8.3 \times 10^{33} \times 6.901}{2.015 \times 9.55 \times 10^{11}}=1.982 \times 10^{15} .
$$

On the other hand it is known that [1]

$$
\begin{aligned}
& 1-\beta=0.283 \\
& \beta=0.717 ;
\end{aligned}
$$

in such way that the magnitude of the self-generated magnetic field in the center of the star is, according to (65)

$$
H_{o}=\left[\frac{2 \times 3.14 \times 0.2446 \times 10^{15} \times 0.283}{0.717}\right]^{1 / 2}=(6.063)^{1 / 2} \times 10^{7} ;
$$

in such way that

$$
H_{o}=2.46 \times 10^{7} \text { gauss . }
$$

Consider another point inside the star, like for example the line $z=3.5$ of the table. From (66) it is easy to see that

$$
\frac{Z}{R^{\prime}}=\frac{3.5}{6.901}=0.507 \text {. }
$$

The point considered is found situated a little further from half the center of the star. From column sixth of the table we have that

$$
\frac{M(r)}{M}=\frac{1.8203}{2.0150}=0.90 \text {. }
$$

In other words, with that choice $90 \%$ of the star mass is being taken into account. The gravitational potential at that point is

$$
\phi=\frac{6.66 \times 10^{-8} \times 8.3 \times 10^{33} \times 3.5}{1.8203 \times 9.55 \times 10^{11}}=1.113 \times 10^{15} .
$$

It is obtained from the third column of the table that

$$
\rho=0.02109 \rho_{o}=0.00260 \mathrm{gr} \cdot \mathrm{cm}^{-3}
$$

and

$$
\rho \phi=2.6 \times 10^{-3} \times 1.113 \times 10^{15}=2.8938 \times 10^{12} .
$$

The magnetic field at that point has the following absolute value

$$
H=\left[\frac{6.28 \times 2.8938 \times 10^{12} \times 0.283}{0.717}\right]^{1 / 2}=(7.1729)^{1 / 2} \times 10^{6}
$$

Namely

$$
H=2.68 \times 10^{6} \text { gauss }
$$


If $H$ and $H_{o}$ are compared, it can be seen that

$$
H=0.11 H_{o} ;
$$

which means that the magnitude of the magnetic field at the middle part of the star has been reduced to $11 \%$ of the value that it has in the center. Since due to boundary condition $\phi=0$ is always taken on the surface, in that region the magnetic field must be calculated with the Formula (20). That way it is obtained that on the surface

$$
H_{s}=6.8 \text { gauss }
$$

With these three points it is possible to build Figure 1 showing the general behaviour of the field.

Consider the case of the Sun, for which we have the following data

$$
\begin{aligned}
& M_{\odot}=1.985 \times 10^{33} \mathrm{gr} \\
& R_{\odot}=6.951 \times 10^{10} \mathrm{~cm} \\
& 1-\beta=0.0498 \\
& \beta=0.9502 \\
& \rho_{m}=1.414 \mathrm{gr} \cdot \mathrm{cm}^{-3} . \\
& \rho_{o}=76.9 \mathrm{gr} \cdot \mathrm{cm}^{-3}
\end{aligned}
$$

In the center of the Sun the gravitational potential has the following value

$$
\phi_{o}=\frac{6.66 \times 10^{-8} \times 1.985 \times 10^{33} \times 6.901}{2.015 \times 6.951 \times 10^{10}}=6.51 \times 10^{15} ;
$$

and then

$$
\phi_{o} \rho_{o}=5.01 \times 10^{17} .
$$

The central magnetic field has a magnitude equal to

$$
H_{o}=4.06 \times 10^{8} \text { gauss . }
$$

Consider the same point used in the above calculation in such way that

$$
\rho=1.62 \mathrm{gr} \cdot \mathrm{cm}^{-3} \text {. }
$$

In that position the gravitational potential has the following value

$$
\phi=3.66 \times 10^{15},
$$

and of course

$$
\rho \phi=5.93 \times 10^{15} .
$$

Besides,

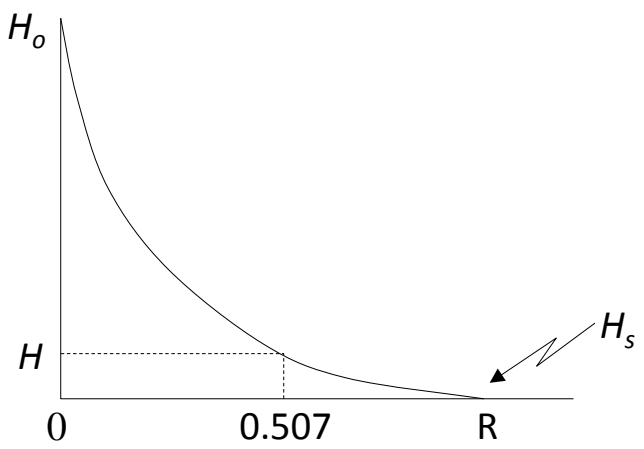

Figure 1. Capella’s magnetic field. 


$$
\frac{1-\beta}{\beta}=0.0524
$$

In consequence

$$
H=4.4 \times 10^{7} \text { gauss . }
$$

Comparing $H$ to $H_{o}$ we have that for the case of the Sun

$$
H=0.11 H_{o} .
$$

As it was expected, the magnitude of the solar magnetic field diminishes in the same proportion as Capella's magnetic field. Indeed, its value is again equal to the $11 \%$ of the one it has in the center. Consequently the corresponding graphic is equal to that of the previous example, as it is easy to see in Figure 2.

The behavior of the magnetic field is in general very similar to the behavior observed in the density of the stellar mass in A.S. Eddington's theory [1] [4].

From the theoretical scheme developed in the above paragraph, it is possible to derive an expression to calculate the average value of the residual magnetic field which acts in the Cepheid type variable stars, in the maximum gravitational compression as well as in that of maximum expansion. If the absolute value of total force is considered, of the equations (45) and (47) as well as the mass-luminosity relation (38), it is possible to demonstrate that in $r=R$

$$
\bar{H}=[8 \pi G M(1-\beta)]^{1 / 2} \cdot\left[\frac{(1-\beta) \bar{\rho}}{\beta(2-\beta)}\right]^{1 / 2} \cdot\left(\frac{1}{R}\right)^{1 / 2}
$$

where the bar indicates the average values of those quantities; $H$ is the residual magnetic field absolute value, $\rho$ the mass density and $R$ the star radius. Besides, the first of the definitions (30) was used. On the other hand, from the result (51) it is easy to see that

$$
\left[\frac{(1-\beta) \bar{\rho}}{\beta(2-\beta)}\right]^{1 / 2}=\frac{6.87 \times 10^{3}}{\tau}
$$

in such way that in (70) the following is obtained

$$
\bar{H}=\frac{\theta}{\tau R^{1 / 2}}
$$

where

$$
\theta=8.89 \times[M(1-\beta)]^{1 / 2}
$$

is a constant which depends on the basic parameters of the particular star being studied.

Therefore, it is possible to state that for each particular Cepheid, the residual magnetic field is inversely proportional to the product of the period and the square root of the radius; so that for the situation of maximum compression we have that

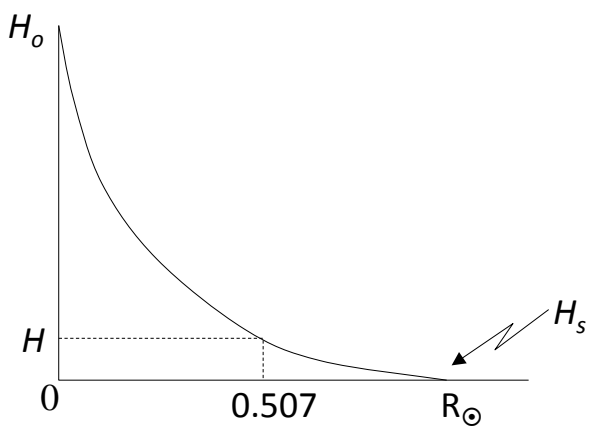

Figure 2. Sun's magnetic field. 


$$
\bar{H}_{\max }=\frac{\theta}{\tau R_{\min }^{1 / 2}}
$$

whereas for the maximum expansion

$$
\bar{H}_{\min }=\frac{\theta}{\tau R_{\max }^{1 / 2}}
$$

The maximum and minimum values of the radius reached by the star in its extreme volumes can be calculated from the relation (25) and from the observational data obtained for the luminosity and the effective temperature at the position of maximum and minimum amplitude of the harmonic oscillations carried out by the particular Cepheid being observed.

\section{Conclusions}

In the present paper, a fundamental hypothesis is made which consists of assuming that all gaseous stars at some early stage of their evolution, and in a natural way, generate an intense magnetic field whose magnitude diminishes from the center of the star to the surface of it in a very peculiar manner. This field generates in the conducting stellar fluid, a magnetic viscosity which gives it a great rigidity that can be interpreted as a rigid superstructure formed by the magnetic lines of force, whose mission is to keep the shape of the star.

That internal magnetic superstructure prevents the star from collapsing gravitationally or exploding because of the added effect of radiation pressures and those of hot gases. In some way, the magnetic lines of force of the self-generated magnetic field behave in the inner part of the star as a powerful shock absorber acting in both ways: against gravitational compression as well as against hot gases and radiation expansion.

When the magnetic field is introduced in the theory in the form of a magnetic hydrostatic pressure [5]-[7], the results previously obtained by Eddington are slightly but substantially modified [1], particularly, it is reduced to half the value of Eddington limit for $k$ [1].

With the help of this result, it is easy to point out the important role the modified limit has in the problem of the stars age and at the same time to indicate a possible solution to the paradox consisting of the existence of stars that are apparently older than the Universe itself. The present theoretical frame derives a simple formula to calculate the magnitude of the average magnetic field on the surface of any gaseous star for which its effective temperature is known.

Since the behavior of the self-generated magnetic field follows a different law in the inner part of the star, a formula is deduced which shows that this field varies like the square root of the product of mass density and the gravitational potential, in both, the center of the star as well as in inner homologous points. Numerical calculations are carried out and the results are presented and then drawn for two specific cases: that of Capella's brightest component and that of the sun.

As it is easy to see, the graphs are exactly alike; which shows that the magnetic field self-generated by the gaseous stars, has the same general behavior for all of them.

Another interesting result refers to the solution of the problem of the Cepheid type variable stars. For those cases, it is proposed that the magnetic field is much weakened due to the fact that those stars are in their last evolutionary stage. However, it is not so much so as not to be able to stop the gravitational collapse acting as a spring which is compressed when the star reaches a certain minimal size. From that minimal volume, the residual magnetic field and the sum of radiation pressures and those of hot gases, inflate the star up to a certain maximum volume from which the compression-expansion cycle is again initiated.

Given the regularity of the oscillations, it is assumed that the process is simple harmonic with a perfectly determined period. Integrating the fundamental result of the theory, which is the relation (11), an ordinary differential equation of simple harmonic oscillator and the expression for the oscillations period in terms of the basic parameters of the star are obtained. In perfect agreement with the observations carried out by other researchers [3] [16] and from the mass-luminosity relation (38), it is shown that the product of the period and the square root of the mean density is equal to a constant.

The numerical value of the constant depends on the star being studied, and it is easily obtained from the theory. It is important to make clear that the above relation is empirically proposed and recorded in the specialized literature as a fact perfectly well established in the different models that on the stars internal structure have been proposed [1] [3] [16]. 
Suppose that the gravitational collapse suffered by a star that is in the last stage of its evolution is so fast and vigorous that the magnetic field noticeably weakened does not have any more an intensity powerful enough so as to stop it initiating the rebound. Under these conditions, the star collapses, and due to the combined effect of material and radiation pressures as well as the sudden hydrogen combustion still present in large amounts in the regions of the star atmosphere, a huge explosion is produced which can send into the space up to 9/10 parts of the star total mass. In that case, it is said that a nova or a supernova has risen depending on the magnitude of the sidereal catastrophe. Whether one thing or another should occur possibly depends on how massive the star is.

The final outcome of the catastrophe will also depend on that last condition. It is possible to have a recurrent nova, a white dwarf, a pulsar, or even a black hole.

\section{References}

[1] Fierros Palacios, A. (2003) The Sunspots. To Be Publishing.

[2] Lane, J.H. (1870) On the Theoretical Temperature of the Sun. A. J. Sci. \& Arts, 4.

[3] Unsöld, A. (1979) El Nuevo Cosmos. Siglo Veintiuno Editores, S.A. México.

[4] Gamow, G. (1967) Una estrella llamada Sol. Espasa Calpe, S.A. Madrid.

[5] Fierros Palacios, A. (1999) El principio tipo Hamilton en la dinámica de los fluidos. Segunda edición, McGraw-Hill, México.

[6] Landau, L.D. and Lifshitz, E.M. (1960) Electrodynamics of Continuous Media. Addison-Wesley Publishing Co., London, England.

[7] Jackson, J.D. (1962) Classical Electrodynamics. John Wiley \& Sons Inc., New York, London.

[8] Cowling, T.G. (1968) Magnetohidrodinámica. Editorial Alhambra, S.A., Madrid, Buenos Aires.

[9] Fierros Palacios, A. (2006) The Hamilton Type Principle in Fluid Dynamics. Fundamentals and Applications to Magnetohydrodynamics, Thermodynamics, and Astrophysics. Springer-Verlag, Wien.

[10] Chandrasekhar, S. (1958) An Introduction to the Study of Stellar Structure. Dover Publications, Inc., New York.

[11] Goldstein, H. (1959) Classical Mechanics. Addison-Wesley Publishing Co., Inc., London, England.

[12] Shwarzschild, M. (1965) Structure and Evolution of the Stars. Dover Publications, Inc., New York.

[13] Landau, L.D. and Lifshitz, E.M. (1959) Fluid Mechanics. Addison-Wesley Publishing Co., London, England.

[14] Werh, M.R. and Richars Jr., J.A. (1960) Physic of the Atom. Addison-Wesley Publishing Co., Inc., London, England.

[15] Fowles, G.R. (1962) Analytical Mechanics. Holt, Rinhart and Winston, New York, Chicago, San Francisco, Toronto, London. http://dx.doi.org/10.1119/1.1941872

[16] Rosseland, S. (1949) Pulsation Theory of Variable Stars. Clarendon Press, Oxford.

[17] Morse, P.M. and Feshbach, H. (1953) Methods of Theoretical Physics. McGraw-Hill Book Company, New York, Toronto, London.

[18] Babcock, H.W. and Cowling, T.G. (1953) M.N., 113, 357.

[19] Parker, E.N. (1955) Hidromagnetic Dynamo Models. The Astrophysical Journal, 122, 293-314. http://dx.doi.org/10.1086/146087

[20] Cowling, T.G. (1981) The Present Status of dynamo Theory. Annual Review of Astronomy and Astrophysics, 19, 115-135. http://dx.doi.org/10.1146/annurev.aa.19.090181.000555

[21] Larmor, J. (1919) Brit. Assoc. Reports.

[22] Cowling, T.G. (1934) Monthly Notices Roy. Astron. Soc. http://dx.doi.org/10.1093/mnras/94.1.39

[23] Stacey, D.F. (1977) Physics of the Earth. 2nd Edition, John Wiley \& Sons, New York, Santa Barbara, London, Sydney, Toronto. 


\section{Appendix I}

Equation (1) which governs the magneto mechanical equilibrium can be used to calculate some formulas by means of which it is possible to estimate pressure, temperature and the magnetic field magnitude orders in the center of any gaseous star in terms of its relevant parameters. Consider then that it is possible to draw within the star, a radius sphere $r$ which contains an amount of mass equal to $M(r)$. When the result (5) is used in (1), that relation is transformed into the following

$$
\mathrm{d}\left(p-\frac{H^{2}}{8 \pi}\right)=-\rho \frac{G M(r)}{r^{2}} \mathrm{~d} r .
$$

From the relation of equivalence (18) and from the first of the definitions (30), it is easy to obtain in (A-1) that

$$
\mathrm{d} p=-\rho \frac{G M(r)}{\beta} \frac{\mathrm{d} r}{r^{2}} .
$$

In order to simplify the calculation, consider that the surface of the sphere is located in the middle region between the center of the star and its boundary, in such way that $M(r) \approx M / 2$, with $M$ the total mass of the star. For $\mathrm{d} p$, the difference between the central whole pressure and the pressure on the surface will be taken, and the latter will be considered as zero.

$\mathrm{Be},-\mathrm{d} r / r^{2} \approx 2 / R$ with $R$ the star radius. In that case from (A-2) the following result is obtained

$$
p_{c}=\frac{\rho_{c} \phi_{c}}{\beta}
$$

where subscript $c$ refers to the star center and

$$
\phi_{c}=\frac{G M}{R}
$$

is the gravitational potential. Central mass density is obtained from the following ratio [1]

$$
\frac{\rho_{c}}{\rho_{m}}=54.36
$$

with $\rho_{m}$ the average mass density.

From the relation (A-3) and from the second of the definitions (30) the following result is obtained

$$
T_{c}=\frac{\mu \phi_{c}}{R}
$$

where $\mu$ is the average molecular weight, $\mathrm{R}$ the gases universal constant and thermal equation of the ideal gas (39) was used.

Finally, in order to obtain the formula which allows estimating the magnitude of the magnetic field, the relation of equivalence (18) and the first of the definitions (30) are used in the Formula (A-3). Thus, for each particular case

$$
H_{c}=\operatorname{constant}\left(\rho_{c} \phi_{c}\right)^{1 / 2}
$$

where

$$
\text { constant }=2 \times\left[\frac{2 \pi(1-\beta)}{\beta}\right]^{1 / 2} .
$$

It will be considered as first example the brightest component of Capella's binary system. Its relevant data are the following

$$
\begin{aligned}
& M=8.30 \times 10^{33} \mathrm{gr} \\
& R=9.55 \times 10^{11} \mathrm{~cm} \\
& \mu=2.2 \\
& \beta=0.717 \\
& 1-\beta=0.283
\end{aligned} .
$$


Besides we have that

$$
\begin{gathered}
G=6.66 \times 10^{-8} \mathrm{ergs} \cdot \mathrm{cm} \cdot \mathrm{gr}^{-2} \\
R=8.26 \times 10^{7} \mathrm{ergs} \cdot \mathrm{mol} \cdot \mathrm{gr}^{-1} \cdot \mathrm{K}^{-1} \\
2 \times\left[\frac{2 \pi(1-\beta)}{\beta}\right]^{1 / 2}=3.15 \\
\phi_{c}=5.8 \times 10^{14} \mathrm{ergs} \cdot \mathrm{gr}^{-1} .
\end{gathered}
$$

Since it is also known that $\rho_{m}=0.00227$, we have that

$$
\rho_{c}=0.1234 \mathrm{gr} \cdot \mathrm{cm}^{-3} \text {. }
$$

With all previous numerical data it is easy to obtain the following results

$$
\begin{aligned}
& p_{c}=9.96 \times 10^{13} \text { dyne } \cdot \mathrm{cm}^{-2} \\
& T_{c}=15.4 \times 10^{6} \mathrm{~K} \\
& H_{c}=2.7 \times 10^{7} \text { gauss }
\end{aligned} .
$$

On the other hand and since

$$
\begin{aligned}
& L=4.8 \times 10^{35} \mathrm{ergs} \cdot \mathrm{sec}^{-1} \\
& 4 \pi G c=2.51 \times 10^{4} \mathrm{ergs} \cdot \mathrm{cm}^{2} \cdot \mathrm{gr}^{-2} \cdot \mathrm{sec}^{-1} . \\
& \alpha=2.5
\end{aligned}
$$

we have that

$$
k_{c}=69 \mathrm{~cm}^{2} \cdot \mathrm{gr}^{-1}
$$

As second example consider the case of the Sun for which we have the following data

$$
\begin{aligned}
& M_{\odot}=1.985 \times 10^{33} \mathrm{gr} \\
& L_{\odot}=3.78 \times 10^{33} \mathrm{ergs} \cdot \mathrm{sec}^{-1} \\
& R_{\odot}=6.951 \times 10^{10} \mathrm{~cm} \\
& \beta=0.9502 \\
& 1-\beta=0.0498 \\
& \phi_{c}=2.0 \times 10^{15} \mathrm{ergs} \cdot \mathrm{gr}^{-1} . \\
& \mu=1 \\
& \rho_{m}=1.414 \mathrm{gr} \cdot \mathrm{cm}^{-3} \\
& \rho_{c}=77 \mathrm{gr} \cdot \mathrm{cm}^{-3}
\end{aligned}
$$

Consequently

$$
\begin{aligned}
& p_{c}=1.6 \times 10^{17} \text { dyne } \cdot \mathrm{cm}^{-2} \\
& T_{c}=24.2 \times 10^{6} \mathrm{~K} \\
& H_{c}=4.5 \times 10^{8} \text { gauss } \\
& k_{c}=276 \mathrm{~cm}^{2} \cdot \mathrm{gr}^{-1}
\end{aligned} .
$$

On the other hand, from the absolute bolometric magnitude determined for the intrinsic variable $\delta$ Cepheid [1], the following data are obtained

$$
\begin{aligned}
& L=2.81 \times 10^{36} \mathrm{ergs} \cdot \mathrm{sec}^{-1} \\
& R=2.32 \times 10^{12} \mathrm{~cm} .
\end{aligned}
$$

Next, to calculate its mass, the mass-luminosity relation (38) will be used as follows 


$$
\begin{aligned}
L & =\frac{4 \pi c G M(1-\beta)}{\alpha k_{c} \beta}=\frac{4 \pi c G M(1-\beta)}{\alpha k_{1} \beta} \frac{T^{3}}{\rho} T_{c}^{1 / 2} \\
& =\frac{4 \pi c G}{\alpha k_{1}} \cdot \frac{3 R}{a \mu} \cdot\left(\frac{G}{4 R} \cdot \frac{R^{\prime}}{M^{\prime}} \frac{\mu}{R}\right)^{1 / 2} M^{3 / 2} \frac{(1-\beta)^{2}}{\beta^{2}} ;
\end{aligned}
$$

where the relations (34), (40) and (59) were used. From the equation [1]

$$
1-\beta=C M^{2} \mu^{4} \beta^{4}
$$

with

$$
C=\frac{\pi G^{3} a}{48 R^{4} M^{\prime 2}}
$$

a constant, mass $M$ can be eliminated. In that case, it is easy to see that

$$
M^{3 / 2}=\left(\frac{48 R^{4} M^{\prime 2}}{\pi G^{3} a}\right)^{3 / 4} \frac{(1-\beta)^{3 / 4}}{\mu^{3} \beta^{3}},
$$

in such way that

$$
L=\frac{4 \pi c G}{\alpha k_{1}} \cdot \frac{3 R}{a} \cdot\left(\frac{G}{4 R} \cdot \frac{R^{\prime}}{M^{\prime}}\right)^{1 / 2} \cdot\left(\frac{48 R^{4} M^{\prime 2}}{\pi G^{3} a}\right)^{3 / 4} \frac{(1-\beta)^{11 / 4}}{\mu^{7 / 2} R^{1 / 2} \beta^{5}} .
$$

With constant values we have that

$$
L=\frac{1.443 \times 10^{71}(1-\beta)^{11 / 4}}{\alpha k_{1} R^{1 / 2} \mu^{7 / 2} \beta^{5}} .
$$

For $k_{1}$ the value found for the brightest component of Capella's binary system will be used [1] and it will be taken for the average molecular weight $\mu$ the usual numerical value 2.11 [1]. Since $\beta$ is the only unknown quantity in the above relation; the equation obtained finally is

$$
(1-\beta)^{11 / 4}=0.909 \beta^{5}
$$

From its numerical solution the following data are obtained

$$
\begin{aligned}
& \beta=0.545 \\
& 1-\beta=0.455 \\
& 2-\beta=1.455 .
\end{aligned}
$$

Using those values in the quadric Equation (32) it is easily obtained that

$$
M=1.82 \times 10^{34} \text { gr . }
$$

On the other hand and since the volume is

$$
V=5.23 \times 10^{37} \mathrm{~cm}^{3}
$$

the mean density of the intrinsic variable $\delta$ Cepheid has the following value

$$
\rho_{m}=3.5 \times 10^{-4} \mathrm{gr} \cdot \mathrm{cm}^{-3}
$$

To conclude, it is easy to prove that using the relation of equivalence (18) and the formulas (30) and (26), the following equation is obtained

$$
H=\left[\frac{8 \pi R \varrho T(1-\beta)}{\mu \beta}\right]^{1 / 2}
$$

by means of which it is also possible to calculate the magnitude of the self-generated magnetic field, in both, the center as well as the inner homologous points of any gaseous star. 
From the results of paragraph 7 and with the help of numerical data from the table, from relations (59) and (67) it is obtained for the Capella case that

$$
\begin{aligned}
& T_{c}=13.2 \times 10^{6} \mathrm{~K} \\
& \rho_{c}=0.1234 \mathrm{gr} \cdot \mathrm{cm}^{-3} ;
\end{aligned}
$$

In such away that in the center of the star, the magnitude of the self-generated magnetic field is again

$$
H_{c}=2.46 \times 10^{7} \text { gauss . }
$$

From the second column of the table we have that for $z=3.5, u=0.27629$ so that at this point

$$
\begin{aligned}
& T=3.65 \times 10^{6} \mathrm{~K} \\
& \rho=2.6 \times 10^{-3} \mathrm{gr} \cdot \mathrm{cm}^{-3} \\
& H=2.0 \times 10^{6} \text { gauss. }
\end{aligned}
$$

Likewise, for the center of the Sun the following results are obtained

$$
\begin{aligned}
& T_{c}=19.7 \times 10^{6} \mathrm{~K} \\
& \rho_{c}=77 \mathrm{gr} \cdot \mathrm{cm}^{-3} \\
& H_{c}=4.06 \times 10^{8} \text { gauss. }
\end{aligned}
$$

It can be proven that in the point $z=3.5$,

$$
\begin{aligned}
& T=5.4 \times 10^{6} \mathrm{~K} \\
& \rho=1.62 \mathrm{gr} \cdot \mathrm{cm}^{-3} \\
& H=3.1 \times 10^{7} \text { gauss. }
\end{aligned}
$$

It is easy to see that for the calculated values of $H$ and $H_{c}$ with this last method, it is also fulfilled that in both examples the ratio $H / H_{c}$ always keeps the same proportion. In other words, it can be proved that

$$
H=\left(u^{n+1}\right)^{1 / 2} H_{c} .
$$

The numerical values of $u^{n+1}$ can be looked up on the fourth column of the table for different points within the star.

From the Equations (20), (22), (25) and from the mass-luminosity relation (38), the following expression to calculate the mass for any gaseous star, using some of their basic parameters is obtained

$$
M=\left(\frac{\beta k_{c}}{1-\beta}\right) \cdot\left(\frac{\alpha a}{4 m^{2} G}\right) \cdot(R H)^{2}
$$

With the numerical values of the constants, the next result is obtained

$$
\left(\frac{\alpha a}{4 m^{2} G}\right)=1.12 \times 10^{6} \mathrm{gr}^{2} \cdot \mathrm{cm}^{-4} \cdot \text { gauss }^{-2}
$$

Consequently, for a particular star is fulfilled that

$$
M=\text { constant } \times(R H)^{2}
$$

where

$$
\text { constant }=1.12 \times 10^{6}\left(\frac{\beta k_{c}}{1-\beta}\right) .
$$

In other words, the mass of a gaseous star is proportional to the square of the product of its radio and the magnitude that the self-generated magnetic field has on its surface. From the data we have from the Sun it is easy to verify by direct calculations that $M_{\odot}=2.0 \times 10^{33} \mathrm{gr}$; whereas for Capella we have that $M=8.3 \times 10^{33} \mathrm{gr}$. 


\section{Appendix II}

\section{The self-generated magnetic field of gaseous stars.}

Let's consider the case of any gaseous star of radius $R_{*}$, composed by a viscous and conducting compressible gaseous fluid, which from the thermo dynamic viewpoint behaves as an ideal gas. The stellar fluid is insolated in space, at very high temperature and pressure conditions and remains together by its own gravitational attraction and at dynamic equilibrium with the force produced by the sum of the pressures of radiation and the hot gases. The astronomical observations, seems to indicate that in general is under the influence of a poloidal magnetic field $\boldsymbol{B}_{*}(x, t)$; [18]. In addition, the star revolves on its own axis with a velocity $\boldsymbol{v}_{*}\left(\ell_{*}, t\right)$, with $\ell_{*}$ the stellar latitude.

In order to determine the dynamic state of a viscous and conducting compressible gaseous fluid, that moves with velocity $\boldsymbol{v}(x, t)$ in some region of space where a magnetic field $\boldsymbol{H}(x, t)$ exist, the following equations are used

$$
\operatorname{div} \boldsymbol{H}=0
$$

and

$$
\begin{aligned}
\frac{\partial \boldsymbol{v}}{\partial t}+(\boldsymbol{v} \cdot \operatorname{grad}) \boldsymbol{v}= & -\frac{1}{\rho} \operatorname{grad} p+\frac{\eta}{\rho} \nabla^{2} \boldsymbol{v}+\frac{1}{\rho}\left(\zeta+\frac{1}{3} \eta\right) \operatorname{grad} \operatorname{div} \boldsymbol{v} \\
& -\frac{1}{4 \pi \rho}(\boldsymbol{H} \times \boldsymbol{r o t} \boldsymbol{H}) ;
\end{aligned}
$$

where $p(x, t)$ is the total pressure, $\rho(x, t)$ the mass density and $\eta$, $\zeta$ the coefficients of viscosity [6] [9]. The relation (B-2) is the momentum balance equation of magnetohidro dynamics (MHD). For any gaseous star, the mass density is in general, a function of time $t$ and the radius $R_{*}$, such that, $\rho_{*}=\rho\left(R_{*}, t\right)$.

It can be directly shown that (B-2) takes the following equivalent form

$$
\frac{\partial \boldsymbol{v}}{\partial t}+(\boldsymbol{v} \cdot \operatorname{grad}) \boldsymbol{v}+\frac{1}{\rho_{*}} \operatorname{grad}\left(p+p_{r}\right)=\frac{1}{\rho_{*}} \frac{\partial}{\partial x^{j}}\left[\sigma_{i j}^{\prime}+\frac{H_{i} H_{j}}{4 \pi}\right]
$$

where $\tilde{\sigma}^{\prime}(x, t)$ is the viscosity stress tensor [6] [9], the relation of equivalence (18) and the following well known formula in vector analysis

$(\boldsymbol{H} \cdot \operatorname{grad}) \boldsymbol{H}=\boldsymbol{g r a d}\left(H^{2} / 2\right)-\boldsymbol{H} \times \boldsymbol{r o t} \boldsymbol{H}$ were used; after integration by parts and taking into account the condition (B-1). Suppose that the star revolves in such a way that the flux of the stellar fluid is steady, and then $\partial \boldsymbol{v}_{*} / \partial t=0$. In this case, it is fulfilled that $\left(\boldsymbol{v}_{*} \cdot \operatorname{grad}\right) \boldsymbol{v}_{*}=\operatorname{grad}\left(v_{*}^{2} / 2\right)-\boldsymbol{v}_{*} \times \boldsymbol{\operatorname { r o t }} \boldsymbol{v}_{*}=0$; because $\boldsymbol{v}_{*}$ is independent of $x$.

Using this last result in (B-3) and with the help of Equations (9), (19) and (39), it can be directly obtained that

$$
H_{*}^{2}=\frac{4 \pi \rho_{*} R T_{*}}{\mu}+\frac{8 \pi a}{3} T_{* e}^{4}
$$

because $\operatorname{div} v\left(\ell_{*}, t\right)=0$. This last result is valid for any gaseous star with radius $R_{*}$, that revolves with a rotational velocity $\boldsymbol{v}_{*}\left(\ell_{*}, t\right)$. On the other hand is easy to see that in the inner regions of a given star, the first term is greatest than the second one, and therefore that term can be neglected.

Consequently and for any inner region of the star, the magnitude of the magnetic field can be calculated from the following equation

$$
H_{*}=\left[\frac{4 \pi \rho_{*} R T_{*}}{\mu}\right]^{1 / 2} .
$$

At the surface of the star, the mass density is practically zero, and then, the magnitude of the magnetic field can be obtained from the next relation

$$
H_{* s}=m T_{* e}^{2}
$$


which is Equation (20) of the text. Let's, consider that $H_{*}$ is the magnitude of the self-generated magnetic field by gaseous stars. Then, it is easy to see that in the inner regions of gaseous stars, the self-generated magnetic field varies like the square root of the product of the mass density and the absolute temperature; both calculated at those regions. On the other hand, at the surface, it depends on its effective temperature; according to different laws perfectly established.

In the theoretical frame of MHD and because the magnetic permeability of the media differs only slightly from unity, and the difference is unimportant [6] [9], it is consider that, $\boldsymbol{H}=\boldsymbol{B}_{*}$ and then, the self-generated magnetic field fulfill the basic laws of magneto statics; that in their differential form are the condition (B-1) and the following relation

$$
\operatorname{rot} B_{*}=\frac{4 \pi}{c} j
$$

where $\boldsymbol{j}$ is the steady-state current distribution localized in some region of the convective zone and $c$ is the velocity of light in empty space. According to (B-1), $\boldsymbol{B}_{*}(\boldsymbol{x})$ must be the curl of some vector field $\boldsymbol{A}_{*}(\boldsymbol{x})$, called the vector potential [7]; that is,

$$
B_{*}(x)=\operatorname{rot} A_{*}(x)
$$

For a steady-state current distribution localized in a relative small region of space, the vector potential is given by the following expression [7]

$$
A_{*}(x)=\frac{1}{c} \int \frac{j\left(x^{\prime}\right)}{\left|x-x^{\prime}\right|} \mathrm{d}^{3} x^{\prime}
$$

where $\boldsymbol{x}^{\prime}$ is a distance measured relative to a suitable origin in the localized current distribution [7]. Starting with (B-8) it expands the denominator in powers of $\boldsymbol{x}^{\prime}$, until the lowest order of approximation. Then for a given component of the vector potential will have the next expansion [7]

$$
A_{* i}(x)=\frac{1}{c x} \int j_{i}\left(x^{\prime}\right) \mathrm{d}^{3} x^{\prime}+\frac{x}{c x^{3}} \cdot \int j_{i}\left(x^{\prime}\right) x^{\prime} \mathrm{d}^{3} x^{\prime}+\cdots ;
$$

where $\boldsymbol{x}$ is the coordinate of a point at a great distance of the localized current distribution. The fact that $\boldsymbol{j}$ is a localized divergenceless current distribution, allows simplification and transformation of expansion (B-9). Let $f\left(\boldsymbol{x}^{\prime}\right)$ and $g\left(\boldsymbol{x}^{\prime}\right)$ be well-behaved functions of $\boldsymbol{x}^{\prime}$. Then, if $\boldsymbol{j}\left(\boldsymbol{x}^{\prime}\right)$ is localized and has zero divergence, it has [7]

$$
\int\left(f \boldsymbol{j} \cdot \operatorname{grad}^{\prime} g+g \boldsymbol{j} \cdot \boldsymbol{g r a d} f\right) \mathrm{d}^{3} x^{\prime}=0 .
$$

This can be established by an integration by parts of the second term, followed by expansion of $f d_{i v^{\prime}} \cdot(g j)$. With $f=1$, and $g=x_{i}^{\prime}$, the last expression establishes that

$$
\int j_{i}\left(x^{\prime}\right) \mathrm{d}^{3} x^{\prime}=0 .
$$

The first term in (B-9) corresponding to the monopole term in the electrostatic expansion is therefore absent. The integral in the second term of (B-9) can therefore be written in the following form

$$
\boldsymbol{x} \cdot \int \boldsymbol{x}^{\prime} j_{i} \mathrm{~d}^{3} x^{\prime}=-\frac{1}{2}\left[\boldsymbol{x} \times \int\left(\boldsymbol{x}^{\prime} \times \boldsymbol{j}\right) \mathrm{d}^{3} x^{\prime}\right]_{i} .
$$

It is customary to define the magnetic moment density or magnetization as [7]

$$
\mathcal{M}(\boldsymbol{x})=\frac{1}{2 c}[\boldsymbol{x} \times \boldsymbol{j}(\boldsymbol{x})],
$$

and its integral as the magnetic moment $m$; that is

$$
\boldsymbol{m}=\frac{1}{2 c} \int \boldsymbol{x}^{\prime} \times \boldsymbol{j}\left(\boldsymbol{x}^{\prime}\right) \mathrm{d}^{3} x^{\prime}
$$


Then, the vector potential from the second term in (B-9) is the magnetic dipole vector potential

$$
A_{*}(x)=\frac{m \times x}{x^{3}} .
$$

This is the lowest nonvanishing term in the expansion of $\boldsymbol{A}_{*}$ for a localized steady-state current distribution. The corresponding magnetic induction $\boldsymbol{B}_{*}$ can be calculated directly by evaluating the curl of the last equation [7]; that is

$$
\boldsymbol{B}_{*}=\frac{3 \boldsymbol{n}(\boldsymbol{n} \cdot \boldsymbol{m})-\boldsymbol{m}}{x^{3}}
$$

where $\boldsymbol{n}$ is a unit vector in the direction $\boldsymbol{x}$. The magnetic induction $\boldsymbol{B}_{*}$ has exactly the form of the field of adipole. Faraway from any localized steady-state current distribution, the magnetic induction is that of a magnetic dipole of dipole momentum given by (B-11). Additionally, from Equation (B-2) it can be shown that

$$
\boldsymbol{T}=\frac{1}{c} \int\left(\boldsymbol{x}^{\prime} \times\left[\boldsymbol{j} \times \boldsymbol{B}_{*}\right]\right) \mathrm{d}^{3} \boldsymbol{x}^{\prime}
$$

where $\boldsymbol{T}$ is the total torque and only the magnetic part of the force was used and taking into account the Equation (B-6). From this last result and writing out the triple vector product, it can be directly obtained that

$$
\boldsymbol{T}=\frac{1}{c} \int\left[\left(\boldsymbol{x}^{\prime} \cdot \boldsymbol{B}_{*}\right) \boldsymbol{j}-\left(\boldsymbol{x}^{\prime} \cdot \boldsymbol{j}\right) \boldsymbol{B}_{*}\right] \mathrm{d}^{3} x^{\prime} .
$$

The second integral vanishes for a localized steady-state current distribution, as can be seen from equation before (B-9) with $f=g=x^{\prime}$ [12]. The first integral is therefore [12]

$$
\boldsymbol{T}=\boldsymbol{m} \times \boldsymbol{B}_{*}
$$

This is the familiar expression for the torque on a dipole; it's one of the ways of defining the magnitude and direction of the magnetic induction $\boldsymbol{B}_{*}$ [7].

Consequently, the self-generated magnetic field of gaseous stars, is produced by some special kind of mechanism. In fact, according to density and temperature conditions, it can exist some region into the convective zone that has a maximum of ionization.

The electrically charged particles are moved by the convective streams across that region, making its contribution to the steady-state current distribution localized and goon; and they are continuously replaced by other particles.

That is an effect likely in form to the one that produce the rainbow. The drops of water moved across the region where the rainbow is produced, making its contribution to the phenomenon and continuing with its fall; and they are continuously replaced by other drops.

It is for that effect, that the rainbow is a steady-state and localized optical phenomenon; like is too, the proposed steady-state current distribution localized.

Since this current distribution is produced by the high ionization of the region and the process of ionization depends on density and temperature conditions of the region, the self-generated magnetic field of gaseous stars is a function of these variables, as is easy to see form Equation (B-5).

To conclude, it is important to mention what follows: concerning the elaboration of an alternative theoretical scheme on the origin and structure of the magnetic field self-generated by gaseous stars, many researches have engaged themselves, throughout 40 or more years, to the self-exited dynamo models [19].

Unfortunately, the theoretical results obtained by then are far from satisfactory even now [20]. The model was initially proposed in 1919 by J. Larmor [21] with the purpose of giving an explanation to the phenomenon of Sunspots. That suggestion was quickly rejected for being inadequate and inconsistent to the astronomical observations about the phenomenon [22].

Also, by means of this model, the idea is to explain the origin and structure of the magnetic field self-generated by the Earth. Neither in this case it has been possible to give any explanation which is satisfactory concerning the basic characteristic of the above mentioned magnetic field [23]. 


\section{Appendix III}

\section{Calculation of the absolute magnitude.}

The absolute magnitude of any variable star of the Cepheid type can be calculated from the average effective temperature and also from the constant of the period-mass density relation (51). That is to say

$$
\text { constant }=0.08 \times\left[\frac{\beta(2-\beta)}{1-\beta}\right]^{1 / 2} .
$$

The methology is as follows. It is necessary to propose a numerical value for the constant, which will depend on the period measure din days. Generally, it is estimated that the value goes from 0.1 to 0.13 . Thus, the value is near to lower limit if the period is of days, and to the uppler limit if it is of hours. In other words, that value is small if the period is large, and big if it is short. Then, according to the relationship (42)

$$
\rho_{m}^{1 / 2}=\frac{\lambda}{\tau}
$$

where $\lambda$ is the numerical value of the constant, $\tau$ is the period measured in days, and $\rho_{m}$ is the average mass density.

With the use of $\lambda$ in (C-1), a second degree equation is obtained, and from it, the values of $\beta$ and $1-\beta$ can be calculated. Then, for any gaseous star the value of the stellar mass $M$, in terms of the Sun's mass, is determined from the quadric Equation (32). That relation may be solved with the average value of the molecular weight $\mu$ which is generally taken as a constant numerically equal to 2.2 . For a red variable star, $\mu=2.11$. The value of the radius $R$ is given by the following expression in terms of $\rho_{m}$ and $M$

$$
R=\left(\frac{3 M}{4 \pi \rho_{m}}\right)^{1 / 3} .
$$

From the observed average effective temperature and with the help of relationships (20), (21) and (22), it is easier to calculate the magnitude that has at the stellar surface the self-generated magnetic field by the variable star of the Cepheid type. Finally, the absolute magnitude of any variable star of the Cepheid type and also for a red variable, is found directly from the relationship existing between the radius and the self-generated magnetic field at the surface, and with the help of the Sun's parameters, considering it as the unit measure.

$$
\log \left(\frac{R H}{R_{\odot} H_{\odot}}\right)=\frac{M_{b o l \odot}-M_{\text {bol }}}{5.024}
$$

In order to estimated the distance at which the variable star is found, it visual and absolute or bolometric magnitudes are used in the following expression

$$
m_{\text {vis }}-M_{\text {bol }}=5.024 \log \frac{r}{10}
$$

where $r$ is the distance in parsecs. The relationship (C-5) is the distance modulus.

For the long-period variables the period-mass density relation (52), and the last methodology can be used to obtain absolute magnitudes and other data of that class of variables. 